\title{
Behandlung der akuten und chronischen Tendinitis beim Pferd mit lokaler Lasertherapie und Laserakupunktur
}

\author{
Eine Studie an 30 Fållen
}

\author{
Uwe Petermann
}

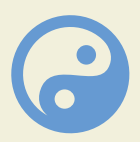

\section{Zusammenfassung}

In dieser Arbeit werden 30 Pferde mit akuter und chronischer Tendinitis der ober-

flächlichen Beugesehne und des Fesselträgers vorgestellt, die ausschließlich mit Laserakupunktur behandelt wurden. Neben der Diskussion des gesamten Patientengutes wird das Vorgehen und die Wirksamkeit der Laserakupunktur mit einem 904 nm Gallium Arsenit Laser bei der Therapie von vollständigen Durchtrennungen der oberflächlichen Beugesehne anhand von 2 Fallstudien demonstriert. Dabei wird die Wichtigkeit der Wahl des optimalen Lasers hervorgehoben, da speziell die Anwendung des 904 nm Impulslasers mit 90 Watt Impuls Spitzenleistung aufgrund seiner physikalischen Eigenschaften, d. h. der hohen Eindringtiefe in traumatisiertes Gewebe ohne eine thermische Reaktion im Gewebe, der Laser der Wahl ist. Diese Laser sind in der Lage, durch direkte ATP Bereitstellung, hohe Peroxid Clearance, Umstrukturierung der Bindegewebstextur und Anhebung der Produktion von Kollagenfasern eine hervorragende Heilungstendenz im traumatisierten Sehnengewebe zu bewirken. Zusätzlich zur lokalen Lasertherapie werden geeignete Akupunkturpunkte zur Verbesserung der Gewebsdemarkation (3E5), des Gewebeaufbaus und der Kollagenfasersynthese (MP2), der Stimulation des Immunsystems (MP4 und 3E5) und zur antiphlogistischen Therapie (Gb41) mit dem gleichen Laser stimuliert. Neben der Laserakupunktur wurden keinerlei adjuvante Therapien wie Antibiotika oder Antiphlogistika angewandt. Die Ergebnisse dieser Studie zeigen, dass diese Therapie bei Patienten mit akuter und chronischer Tendinitis eine sehr effektive Heilmethode darstellt.

\section{Einleitung}

Die akute und chronische Tendinitis gehören zu den wesentlichen Problemen in der Pferdemedizin. Sie machen etwa 10\% aller Lahmheiten beim Pferd aus [18]. Die Ursachen sind vor allem Überlastung durch Übermüdung [21] und auch dem Trainingszustand unangepasste Belastungen im Sport ohne ausreichendes Aufwärmtraining [3]. Hinzu kommen Verletzungen bei übermütigem Verhalten der Pferde auf der Weide und auch im Stall, wobei es zu plötzlichen über die Belastungsgrenze hinausgehenden Überdehnungen der betreffenden Sehnenabschnitte mit entsprechenden unterschiedlich umfangreichen Zerreißungen von Fibrillen kommt [22]. Bei der chronischen Tendinitis kommt es durch eine zeitliche Aneinanderreihung von jeweils kleineren solcher Traumata zu einer dadurch bedingten fortschreitenden Vernarbung der Sehnen [3]. Die Elastizität geht zunehmend verloren, da die Reparaturen der Traumata durch straffes Bindegewebe erfolgen, in das keine kollagenen Fasern eingebettet werden [4]. Das Ergebnis ist, dass die verbleibenden intakten kollagenen Strukturen einem immer größeren Stress ausgesetzt werden und dadurch die Bereitschaft zu weiteren Insulten permanent zunimmt [22].

\section{Therapiemo̊glichkeiten}

Bekannte Therapiemöglichkeiten sind die Hyaluronsäureinjektion [4], Stoßwellentherapie [10], Kohlefaserimplantation [23], Sehnensplitting [9] und die Behandlung mit Stammzellen [2]. Die Ergebnisse dieser Behandlungsmethoden werden von verschiedenen Autoren unterschiedlich beurteilt. Keine dieser Therapieformen scheint aber die Methode der Wahl darzustellen, da sie häufig nicht zu einer vollständigen Institutio ad integrum führen [3]. Daher gilt nach wie vor die Rehabilitation durch konsequente Boxenruhe mit täglichem Schritttraining auf hartem, ebenem Grund als ein wesentlicher Bestandteil der Therapie.

\section{Therapie mit Lasertherapie und Laserakupunktur}

Das Problem bei der akuten und chronischen Tendinitis der oberflächlichen Beugesehnen, des Unterstützungsbandes und des Fesselträgers ist, dass die zerstörten Sehnenfasern bindegewebig repariert werden. Dieses straffe Bindegewebe ist nicht nur unelastisch und daher extrem anfällig für erneute Insulte, sondern auch so kompakt, dass elastische Fasern nicht in ausreichendem Maße penetrieren können, um den ursprünglichen elastischen Zustand der Sehne wieder herzustellen [4]. Die lokale Lasertherapie oder Low Level Laser Therapy (LLLT) spielt daher bei der Tendinitisbehandlung eine ganz wesentliche Rolle, da sie nach meiner Erfahrung eine optimale Möglichkeit darstellt, das straffe Bindegewebe umzustrukturieren und aufzulockern, sodass wieder Kollagenfasern eingelagert werden können und gleichzeitig den Körper bei der Bildung von Kollagenfasern unterstützen. Beide Mechanismen sind nachgewiesen $[11,16]$.

\section{Untersuchungsergebnisse zu den Wirkungsmechanismen des Therapielasers \\ Biophysikalische Grundlagen der Laserwirkung}

Speziell für den Infrarotlaser gibt es eingehende Untersuchungen [24]. Danach werden etwa $70 \%$ der Laserenergie an der Hautoberfläche reflektiert, 15-20\% der eintretenden Energie werden gestreut 
und verlieren sich diffus im Körpergewebe. Es werden also nur 5-10\% der Laserenergie absorbiert. Die Absorption erfolgt über ein sogenanntes „Antennenpigment“" bei dem es sich um ein wichtiges Glied der Atmungskette in den Mitochondrien handelt, das Flavoprotein-Metall-Redoxsystem. Die absorbierten Laserphotonen werden ähnlich der Photosynthese im Blattgrün hier direkt in Zellenergie umgewandelt und führen zu einem direkten Anstieg der ATP-Konzentration im bestrahlten Gewebe [7,25]. Diese Energie kann wiederum direkt zur Bewältigung von Reparaturprozessen, zur Umstrukturierung von pathologischem Gewebe sowie zur Synthese von Kollagenfasern genutzt werden. Ebenso kann ein überstarker Energieschub in den Nervenzellen des Akupunkturpunktes zur Hyperpolarisation und damit zur Abblockung von Reizen führen, womit die erwiesene Schmerzreduktion durch den Laser zu erklären wäre. Diese Untersuchungen wurden bestätigt und fortgeführt durch Hamblin [5] in dem Artikel Mechanisms of Low Level Light Therapy. Michael R. Hamblin ist Associate Professor an der Harvard Medical School und Leiter des The Wellman Center for Photomedicine, der Harvard Medical School und dem Massachusetts Institute of Technology (MIT). Die ersten wichtigen Untersuchungen wurden von Mester [11], dem Pionier der LLLT, bereits 1969 durchgeführt und zeigten nach 1-3 Laserbestrahlungen von Wunden eine Vermehrung von Kollagenfasern und eine starke Zunahme der Zellaktivität. Eine weitere interessante Untersuchung von Karu et al. [8] zeigt, dass Laserlicht die Clearance von Peroxidradikalen erheblich steigern kann, was die enorme heilende Wirkung auf Entzündungen jeglicher Art erklärt.

Mehrere Untersuchungen bestätigen eine Dosis-Wirkungsbeziehung in der Form, dass zu kleine Bestrahlungsdosen keinen positiven Effekt haben. Ab einer minimalen Wirkungsdosis wird dann aber mit zunehmender Dosis bis zu einem Maximum eine ansteigende Wirkung erzielt. Über diese Dosis hinaus wird kein weiterer Effekt mehr erzielt. Weitere Untersuchungen zeigen, dass eine negative, destruierende Wirkung auch bei langen Bestrahlungszeiten (bis zu $30 \mathrm{~min}$ ) mit den empfohlenen Lasern nicht nachzuweisen ist [7].

\section{Laserdosis}

Aufgrund allgemeiner Erfahrung zahlreicher Therapeuten im Humanbereich und meiner eigenen Erfahrung in der Veterinärmedizin kann man davon ausgehen, dass eine optimale Wirkung auf oberflächlich gelegene Strukturen, wie z.B. dem Akupunkturpunkt mit einer Laserleistung von 90 Watt Impulsspitzenleistung (Impulslaser) bei einer Bestrahlungszeit von etwa 20-40 sec liegt. Dies entspricht in Abhängigkeit von der Impulsfrequenz einer applizierten Energie von 0,3 bis 1 Joule. Bei tiefer gelegenen Strukturen, wie bei der Therapie der Tendinitis, muss die Therapiedauer auf etwa 2-3 min pro Punkt erhöht werden. Es ist besonders wichtig, darauf hinzuweisen, dass Sehnenläsionen nur mit einem solchen Impulslaser in ausreichendem Maße therapiert werden, da nur dieser in der Tiefe des Gewebes in einer akzeptablen Therapiezeit ausreichend Laserphotonen in das geschädigte Gewebe transportieren kann. Die applizierte Laserenergie beträgt abhängig von der Impulsfrequenz zwischen 1-10 Joule pro Lokalisation [16].

\section{Resonanzfrequenzen}

Neben der Laserleistung gibt es ein weiteres sehr wichtiges Kriterium für die Wirksamkeit der LLLT, aber auch bei der Reizung der Akupunkturpunkte, die Resonanzfrequenzen. Um eine optimale Resonanz zu unterschiedlichen Gewebearten und Gewebszuständen, z. B. Entzündung, Azidose, zu erreichen, kann der kontinuierlichen Laserlichtemission beim Dauerstrichlaser die spezifische Resonanzfrequenz aufmoduliert werden. Beim Impulslaser wird die Resonanzfrequenz durch die Impulsfrequenz der Lichtimpulse erreicht. Inzwischen gibt es drei wichtige Reihen von Resonanzfrequenzen, die nach Nogier [12], nach Bahr [1] und nach Reininger [17] (\$ Tab. 1, 2 und 3). Die ersten Frequenzen wurden von Nogier gefunden. Die „Nogier“-Frequenzen beziehen sich vor allem auf bestimmte Gewebszustände. So hat die Frequenz A' ihre spezifische Wirksamkeit gegen akute Entzündungen, Frequenz B' bei chronischen Entzündungen und organischen Störungen, die Frequenz C' bei allen orthopädischen Erkrankungen, die Frequenz D' wirkt auf einen Teil der psychischen Störungen. Die Frequenz E' wirkt auf das Nervengewebe und das Rückenmark, die Frequenz F' auf den Zahnbereich und subkortikale Gehirnstrukturen und die Frequenz G' auf den Kortex. Die Bahr Frequenzen beziehen sich mehr auf die vegetativen Reaktionszustände. So hat die Frequenz 1' einen Bezug zum Sympathikus. Sie ist die Frequenz der „tiefen Schicht“ bzw. des „tiefen pathologischen Punktes“ TPP. Das bedeutet, dass es sich um den Ursprungspunkt der Erkrankung, also das primäre Krankheitsgeschehen handelt. Die Frequenz 2' ist die Frequenz der „mittleren Schicht“ bzw. des „mittleren pathologischen Punktes“, also der Folgeerkrankungen aus der „tiefen Schicht“, also des sekundären Krankheitsgeschehens. Die Frequenz 2' hat eine Affinität zum Parasympathikus. Die Frequenz 5' ist die spezielle Frequenz der Kardinalpunkte (besonders wirksame Akupunkturpunkte) und auch lokal sehr intensiv wirkende Frequenz bei Entzündungen. Die Frequenz 6' ist die Frequenz des Lenkergefäßes, die Frequenz 7' die Frequenz des Konzeptionsgefäßes. Eine wiederum völlig andere Idee steht hinter den Resonanzfrequenzen nach Reininger [17]. Er fand je eine spezielle Frequenz mit spezifischer Resonanz zu jedem der 12 Meridiane, mit der man die entsprechenden Meridianpunkte besonders intensiv reizen kann. Man kann mit dieser Frequenz auch die den Meridianen zugehörigen Organe wie zum Beispiel die Lunge, die Leber oder die Niere direkt im Sinne einer LLLT mit der „Lungen-, Leber-, Nierenfrequenz“ bestrahlen.

\section{Material und Methoden Geeignete Laser zur lokalen Lasertherapie der Tendinitis}

Die Art des zur Lasertherapie und Laserakupunktur verwendeten Lasers spielt bei der Behandlung eine fundamentale Rolle, da in der Lasertherapie sehr unterschiedliche Bauarten von Lasern Verwendung finden, die verschiedene Wellenlängen und vor allem sehr unterschiedliche Eindringtiefen in das Gewebe besitzen $[14,15,19]$.

Generell befinden sich zwei Typen von Lasern auf dem Markt, die hierbei unterschieden werden müssen. Zum einen gibt es Dauerstrichlaser, die kontinuierliches Laserlicht aussenden. Die Leistung dieser Geräte liegt zwischen 30 und $200 \mathrm{~mW}$. Die zweite Laserart ist der Impulslaser, bei 
Tab. 1 Laserfrequenzen A'-G' nach Nogier. Quelle: [12].

\begin{tabular}{|c|c|c|}
\hline Frequenz A' & $\begin{array}{l}292 \mathrm{~Hz}\left(\mathrm{~A}^{\prime \prime}\right. \\
37376) \mathrm{Hz}\end{array}$ & $\begin{array}{l}\text { Entzündungsfrequenz, Störherdfrequenz, Gewebsdemarkation, akute } \\
\text { Zustände }\end{array}$ \\
\hline Frequenz B' & $584 \mathrm{~Hz}$ & $\begin{array}{l}\text { Organfrequenz, anabole Frequenz, chronische Zustände, Tonisierungs- } \\
\text { punkte, Zonenfrequenz des Bauchbereiches, Sehnenschäden, Band- } \\
\text { apparat, Kollagensynthese }\end{array}$ \\
\hline Frequenz C' & $1168 \mathrm{~Hz}$ & $\begin{array}{l}\text { Körperfrequenz (Standartfrequenz für fast alle Körperakupunktur- } \\
\text { punkte), Muskeln, Gelenke, Wirbelsäule, }\end{array}$ \\
\hline Frequenz D’ & $2336 \mathrm{~Hz}$ & \\
\hline Frequenz E' & $4672 \mathrm{~Hz}$ & $\begin{array}{l}\text { Rückenmarksfrequenz, nervale Störungen, Neuralgien, Neuritiden, } \\
\text { Lähmungen }\end{array}$ \\
\hline Frequenz F' & $9344 \mathrm{~Hz}$ & Zonenfrequenz des Zahnbereiches, Antidepression, Emotion, Subkortex \\
\hline Frequenz G' & $\begin{array}{l}18688 \mathrm{~Hz} \\
(\mathrm{G} 146 \mathrm{~Hz})\end{array}$ & $\begin{array}{l}\text { Psychosomatischer Hauptpunkt, Kortex, ZNS-Gedächtnispunkte (bei } \\
\text { chronischen Schmerzen) }\end{array}$ \\
\hline Frequenz A" & $37376 \mathrm{~Hz}$ & \\
\hline
\end{tabular}

Tab. 2 Laserfrequenzen 1-7 nach Bahr. Quelle: [1].

\begin{tabular}{|c|c|c|}
\hline Frequenz 1' & $599,5 \mathrm{~Hz}$ & $\begin{array}{l}\text { Frequenz der "tiefen Schicht“ bzw. des „tiefen pathologischen Punktes“ } \\
\text { TPP, primäres Krankheitsgeschehen, Affinität zum Sympathikus }\end{array}$ \\
\hline Frequenz 2' & $1199 \mathrm{~Hz}$ & $\begin{array}{l}\text { Frequenz der „mittleren Schicht“ bzw. des „mittleren pathologischen } \\
\text { Punktes“, sekundäres Krankheitsgeschehen, Affinität zum Parasympa- } \\
\text { thikus }\end{array}$ \\
\hline Frequenz 3' & $2398 \mathrm{~Hz}$ & $\begin{array}{l}\text { Frequenz der „Oberflächenschicht“ auf der lateralen Seite, „, biotische } \\
\text { Punkte“, Vitaminpunkte, Punkte der „Belastungen“ auf der Stirn }\end{array}$ \\
\hline Frequenz 4' & $4796 \mathrm{~Hz}$ & $\begin{array}{l}\text { Frequenz der Oberflächenschicht auf der kontralateralen Seite, } \\
\text { „biotische Punkte“, Mineralstoffpunkte, Spurenelementpunkte }\end{array}$ \\
\hline Frequenz 5' & $9592 \mathrm{~Hz}$ & $\begin{array}{l}\text { „antioszillatorische“ Frequenz, Frequenz der Kardinalpunkte, des MGO } \\
\text { (Ni3) und der PGO (persönliche Punkte gegen Oszillation), lokal bei } \\
\text { Entzündungen }\end{array}$ \\
\hline Frequenz 6' & $\begin{array}{l}19184 \mathrm{~Hz} \\
(149,875 \mathrm{~Hz})\end{array}$ & Frequenz des Lenkergefäßes \\
\hline Frequenz 7’' & $\begin{array}{l}38368 \mathrm{~Hz} \\
(299,75 \mathrm{~Hz})\end{array}$ & $\begin{array}{l}\text { Frequenz des Konzeptionsgefäßes, Frequenz der verdeckten } \\
\text { Zahnstörherde }\end{array}$ \\
\hline
\end{tabular}

dem Lichtimpulse sehr hoher Intensität mit Impulsspitzenleistungen von 90 Watt, aber nur mit einer sehr kurzer Dauer von 200 nsec emittiert werden. Diese Laser sind für die für die Sehnentherapie geeigneten Geräte, da sie auch in der Tiefe des Sehnengewebes ausreichend Photonen applizieren. Die von mir verwendeten Laser sind 90 Watt Gallium Arsenit Laser mit einer Wellenlänge von $904 \mathrm{~nm}$ und $200 \mathrm{nsec}$ Impulsdauer und vorprogrammierten Impulsfrequenzen nach Nogier, Bahr und Reiniger (Physiolaser, Reimers \& Janssen GmbH, Berlin).

Obwohl bedingt durch die 1000 fach höhere Spitzenleistung der Lichtimpulse von fast 100 Watt (gegenüber $100 \mathrm{mWatt}$ beim Dauerstrichlaser) die Gewebsdurchdringung des Laserlichts bei diesen Geräten sehr viel höher ist, konnte ich auch bei hohen Impulsfrequenzen von bis zu $40000 \mathrm{~Hz}$ bei täglichem Gebrauch über 7 Jahre keine Schmerzreaktion und auch keine thermische oder gar koagulierende
Wirkung im Gewebe der Patienten beobachten. Diese schonende, aber dennoch tief ins Gewebe eindringende Applikation des Laserlichtes ist bedingt durch die kurze Dauer der Lichtblitze, die bei den sehr hohen Frequenzen auf 100 bzw. 50 nsec verkürzt wird. Diese Laser sind der Laserklasse 3b zugeordnet. Sie sind der Wirkung der Klasse 4-Laser deutlich überlegen, da die Klasse 4-Laser ausschließlich Dauerstrichlaser sind, deren Leistung aber maximal 3 Watt beträgt. Diese Energie wird jedoch zum größten Teil auf der Hautoberfläche absorbiert, wo es aufgrund der Energiedichte zu Verbrennungen und Koagulation kommen kann, ohne tiefere Gewebsschichten in ausreichendem Maße zu erreichen.

\section{Konkrete Therapie der Tendi- nitis mit Laser Akupunktur}

Für die LLLT der Sehne werden lokal die Frequenzen B und A" angewandt und die Sehne je Lokalisation ca. 1 min (entspricht
Tab.3 Laserfrequenzen nach Reininger. Quelle: [17].

$\begin{array}{ll}\text { Lungenmeridian } & 824 \mathrm{~Hz} \\ \text { Dickdarmmeridian } & 553 \mathrm{~Hz} \\ \text { Magenmeridian } & 471 \mathrm{~Hz} \\ \text { Milz Pankreas Meridian } & 702 \mathrm{~Hz} \\ \text { Herzmeridian } & 497 \mathrm{~Hz} \\ \text { Dünndarmmeridian } & 791 \mathrm{~Hz} \\ \text { Blasenmeridian } & 667 \mathrm{~Hz} \\ \text { Nierenmeridian } & 611 \mathrm{~Hz} \\ \text { Kreislauf Sexualität Meridian } & 530 \mathrm{~Hz} \\ \text { 3 Erwärmer Meridian } & 732 \mathrm{~Hz} \\ \text { Gallenblasenmeridian } & 583 \mathrm{~Hz} \\ \text { Lebermeridian } & 442 \mathrm{~Hz}\end{array}$

Laserfrequenzen nach Reininger, die Meridianfrequenzen

ca. 6 Joule) therapiert. Zusätzliche Akupunkturpunkte sind der „Anabolikumpunkt“ MP2, der „Leberpunkt“ Le8, der „Meisterpunkt für Muskeln und Sehnen“ Gb34, und KS6, der Punkt des Sternalganglions. Bei den Tendinitiden sind ebenfalls häufig Wirbelsäulenprobleme in die Therapie mit einzubeziehen, da eine durch radikuläre Reizzustände der Nervenwurzel bedingte inkorrekte Vordehnung der Sehne [1] Vorbereiter für überlastungsbedingte Rupturen von Sehnenfasern darstellen kann. Deshalb wird bei der Tendinitis immer die Wirbelsäule in die Diagnostik und Therapie mit einbezogen. Die entsprechenden Wirbelsegmente findet man oft sehr deutlich als druckdolente Punkte entlang der Wirbelsäule. Zur Therapie werden bilateral die sogenannten Zustimmungspunkte mit der „orthopädischen" Frequenz C nach Nogier behandelt (30 sec, 0,3 Joule). Mit einer Häufigkeit von über $70 \%$ aus meinem Patientengut sind die Zustimmungspunkte der Leber, Bl18, und der Niere, Bl23, auffällig und werden therapiert. Während die akute Tendinitis nach eigener Erfahrung relativ einfach mit Laser-Akupunktur zu behandeln ist, da hier noch keine Reparationen mit straffem Bindegewebe stattgefunden haben, die es bei der chronischen Tendinitis erst wieder umzustrukturieren gilt, ist die Therapie der chronischen Tendinitis deutlich zeitaufwändiger. Die Therapie der akuten Tendinitis wird 4-mal im Wochenabstand in der oben beschriebenen Weise durchgeführt. Während der Therapie wird Boxenruhe mit täglicher 20minütiger Schrittarbeit verordnet. Eine adjuvante Therapie wird nicht benötigt. 


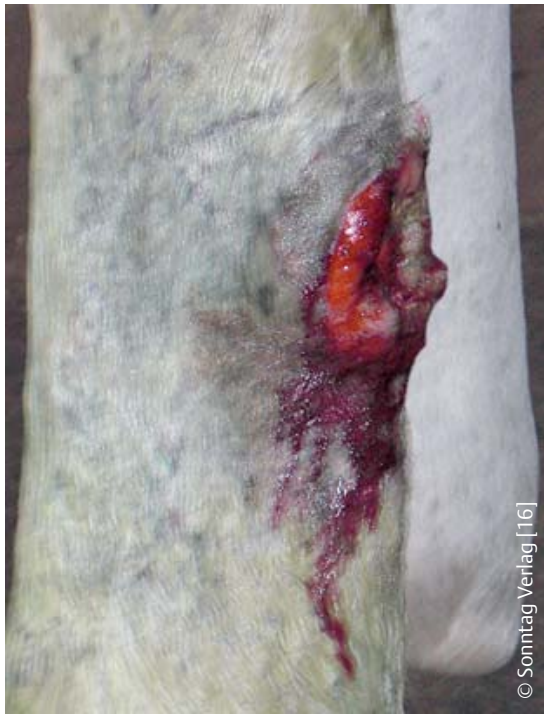

Abb. 1 Infizierte Wunde mit vollständiger Durchtrennung der oberflächlichen Beugesehne.

Danach kann man nach sonografischer Kontrolle mit leichter Schrittarbeit unter dem Reiter mit kurzen Trababschnitten beginnen, um in einem Zeitraum von weiteren 4-6 Wochen die Sehne zu ihrer vollständigen Belastbarkeit zu trainieren.

Die chronische Tendinitis benötigt deutlich mehr therapeutischen Aufwand, da neben der Reparation des eventuellen akuten Schadens hier bereits vernarbte Strukturen wieder umgebaut werden müssen und erst danach die Kollagenfasersynthese erfolgreich stimuliert werden kann, um der Sehne ihre gesunde Struktur zurückzugeben. Die Therapie ist die gleiche wie bei der akuten Tendinitis. Die Behandlungsabstände sind jedoch erheblich kürzer zu gestalten. Bei besonders chronischen, vernarbten Sehnen ist eine stationäre, tägliche Behandlung anzuraten. Nach 2-3 Wochen kann man dann zur ambulanten, wöchentlichen Therapie wechseln, bis die Sehne palpatorisch und sonografisch eine gesunde Textur erkennen lässt. Auch in diesen Fällen ist Stallruhe und tägliche gezielte Bewegung an der Hand wesentlich für die Rehabilitation des Patienten. Die Ausheilungszeit beträgt bei solchen Fällen 10 Wochen bis zu 3 Monaten.

\section{Kasuistik}

Im Folgenden sollen an 2 extremen Beispielen die besonders effektiven Möglichkeiten der Laserakupunktur dargestellt werden.

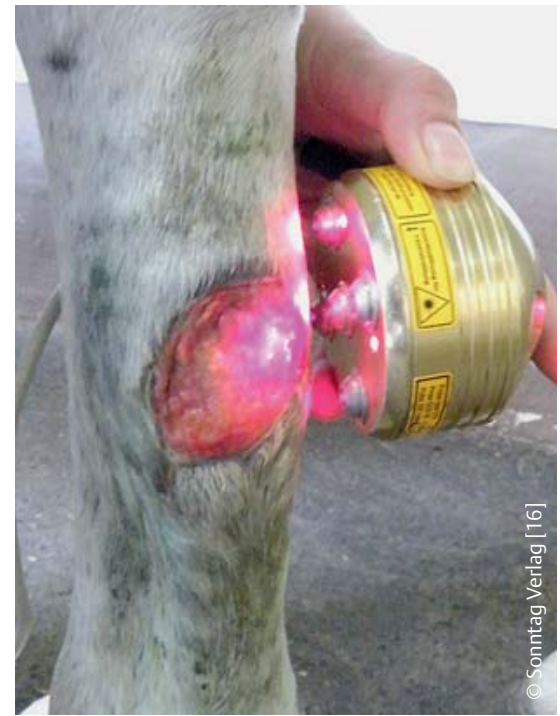

Abb. 2 Therapie der hypergranulierenden Wunde mit einem Flächenlaser ( $5 \times 30$ Watt).

\section{Fallbeispiel 1: Verletzung mit vollständiger Durchtrennung der oberflächlichen Beugesehne}

Eine 17-jährige Hannoveraner Stute (Patient 29) erlitt bei einer Weideverletzung eine vollständige Ruptur der oberflächlichen Beugesehne. In der offenen Wunde konnte man die beiden Enden der Sehne im Abstand von etwa $6 \mathrm{~cm}$ mit der bloßen Hand ertasten und im gebeugten Zustand des Fußes auch in die Wunde vorlagern (๑) Abb. 1).

Da die Wunde für eine chirurgische Versorgung schon zu weit verschmutzt und infiziert war, wurde der Therapie mit Laserakupunktur der Vorzug gegeben, da sowohl die hervorragende gewebsdemarkierende, anti-infektive Wirksamkeit der Methode als auch die besondere Möglichkeit der Wundheilung, speziell der Sehnenheilung, für diese Therapie sprachen.

Die tägliche Behandlung bestand aus einer 5-minütigen Bestrahlung der gesamten Wundfläche, insbesondere der Sehnenenden mit einem starken Impulslaser (Physiolaser Fa. Reimers \& Janssen GmbH, Berlin; Gallium Arsenit Laser $904 \mathrm{~nm}$, 90 Watt Impuls-Spitzenleistung, Impulsdauer 200 nsec). Zur Anwendung kamen die Laserfrequenzen A" und B nach Nogier, $37376 \mathrm{~Hz}$ und $584 \mathrm{~Hz}$. Dies entspricht einer applizierten Laserenergie von 80 Joule. Zusätzlich wurde die Wunde mit einer Laserdusche (Reimers \& Janssen GmbH, Berlin; $5 \times 30$ Watt Multicluster) noch 5 Minuten mit der gewebsaufbauenden Frequenz B bestrahlt (๑Abb. 2).

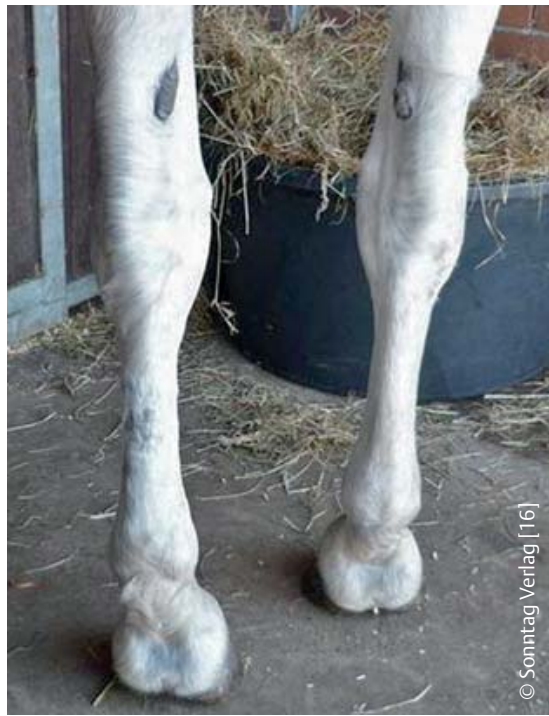

Abb. 3 Vollständig regenerierte Sehne bei Therapieende.

Akupunkturpunkte zur Förderung der Wundheilung (MP2), zur Säuberung und Demarkation des Gewebes (3E5) und zur Entzündungskontrolle (Gb41) wurden ebenfalls täglich mit den entsprechenden Meridianfrequenzen nach Reininger [17] je 30 sec behandelt. Die Wunde wurde zudem täglich mit Wundwasser gesäubert und unter Verband gehalten. Außer einer Tetanus-Auffrischung wurde keine weitere Therapie mit Antibiotika oder Antiphlogistika durchgeführt.

Diese Therapie wurde in der gleichen Weise für 8 Wochen täglich stationär durchgeführt. Danach war die äußere Wunde geschlossen und die Stute wurde entlassen. Die Weiterbehandlung konnte danach durch den Besitzer in der gleichen Weise mit einem baugleichen Lasergerät für 6 Wochen zu Hause weitergeführt und dann abgeschlossen werden. Die Sehne war wieder geheilt und schon fast auf ihre physiologische Stärke zurückgebildet (\$ Abb. 3).

Die Stute wurde im Schritt und zunehmend auch im Trab gearbeitet und wurde nach weiteren 6 Wochen aufbauenden Trainings wieder voll belastet. In einem Beobachtungszeitraum von 3 Jahren war das Pferd lahmfrei und wurde freizeitmäßig Dressur und im Gelände geritten.

Fallbeispiel 2: 8-jährige Hannoveraner Stute mit eiternder Wunde 4 Monate nach OP einer kompletten Durchtrennung der oberflächlichen Beugesehne

Eine 8-jährige Hannoveraner Stute (Patient 28) hatte bei einer Schnittverletzung 
durch einen Weidedraht eine komplette Durchtrennung der oberflächlichen Beugesehne erlitten. Die Sehne war durch eine Drahtnaht adaptiert und anschließend vollständig chirurgisch versorgt worden. 4 Monate nach der Operation der Sehnen war eine eiternde Wunde entstanden, die nicht zur Heilung gebracht werden konnte. Die Hautnaht als auch die Naht der Sehnenscheide zeigten vollständige Nahtdehiszenz. Die Wunde war vollständig mit eitrigem Exsudat verklebt. Die oberflächliche Beugesehne war im Operationsbereich auf einer Länge von etwa $10 \mathrm{~cm}$ daumendick geschwollen. Das Pferd zeigte eine höchstgradige Stützbeinlahmheit im Schritt. Die Therapie wurde in der gleichen Weise (siehe Patient 29) durchgeführt. Verkrampfungen der HWS, BWS und LWS als Folge der langen Schonhaltung wurden über den Punkt Bl10 sowie ebenfalls über die Zustimmungspunkte Bl18 und 23 mit Frequenz $C$ behandelt. Es konnten allerdings nur 2-3 Behandlungen pro Woche durchgeführt werden. Auch bei diesem Patienten wurde die Medikation vollständig abgesetzt, da die Lasertherapie und die Akupunktur für die Kontrolle der Infektion und des Entzündungsgeschehens Sorge tragen würden.

Gut 14 Tage später, nach 5 Behandlungen, war die Wunde geschlossen und die derbe Schwellung der Sehne erheblich zurückgegangen (\$ Abb. 4).

Danach wurden die Sehne und die Akupunkturpunkte für weitere 3 Wochen täglich mit einem 50 Watt Impulslaser von der Besitzerin in gleicher Weise selbst behandelt. Hiernach war die Schwellung und Schmerzhaftigkeit der Sehne nochmals deutlich reduziert (\$ Abb. 5).

Die Stute lief im Trab auf hartem und weichem Boden lahmfrei. Bei einer Nachkontrolle 3 Monate später war kein Unterschied zur gesunden Sehne mehr zu palpieren. Das Pferd wurde wieder voll trainiert und konnte noch mehrere Jahre erfolgreich im Springsport eingesetzt werden.

\section{Ergebnisse}

Es wurden insgesamt 30 Pferde in die Studie einbezogen (\$ Tab.4). Bei den Patienten mit akuter Tendinitis ( $\mathrm{n}=10)$ der oberflächlichen Beugesehne $(n=7)$ und des Fesselträgers $(n=3)$ zeigen sich erwartungsgemäß die besten Resultate. Alle

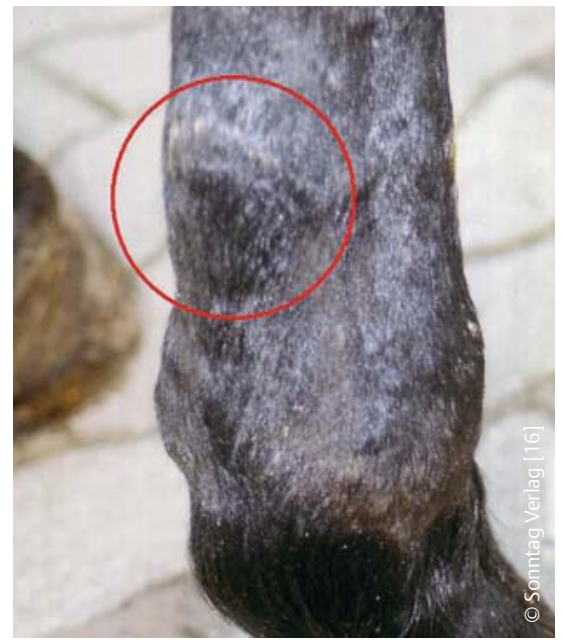

Abb. 4 Sehne nach 5 Behandlungen innerhalb von 14 Tagen. Die stark eiternde Wunde hat sich geschlossen und die Infektion und Schwellung der Sehne hat sich gebessert.

Pferde ( $\mathrm{n}=30)$ konnten nach relativ kurzer Behandlungszeit von 3-6 (im Mittel 4,0) Wochen mit 4-15 Behandlungen (im Mittel 6,1) und weiteren 3 Wochen Rekonvaleszenz als gesund bezeichnet werden und wieder vollständig in ihrem vorherigen Einsatzbereich gearbeitet werden. Es wurden im Beobachtungszeitraum von 3 bis 30 Monaten keine Rezidive beobachtet.

Auch bei den chronischen Tendinitiden der oberflächlichen Beugesehne $(n=8)$ und des Fesselträgers $(n=10)$ war die Therapie sehr erfolgreich. Lediglich ein Patient erlitt ein Rezidiv, als er sich von der Hand losgerissen hatte und in tiefem, sandigen Boden getrabt und galoppiert hatte. Ein weiterer Patient erlitt ein Rezidiv nach 18 Monaten Training und Turniersport und wurde euthanasiert. Alle anderen Patienten konnten ohne Einschränkung die gleiche Arbeit wie vor der Verletzung ausführen ohne erneute Probleme zu bekommen. Interessanterweise konnten auch keine Ausfälle durch andere Krankheiten verzeichnet werden. Es ist zu vermuten, dass dies auch auf den Einfluss der Laserakupunktur-Behandlung zurückzuführen ist, da hiermit immer das gesamte Pferd inklusive etwaiger Rückenerkrankungen oder sonstiger Beschwerden therapiert wird und niemals ausschließlich die Sehne. Sehr auffällig war vor allem die sehr viel höhere Anzahl der Behandlungen, die sich zwischen 3 und 36 Behandlungen belief (im Mittel 15,3), die notwendig waren, um der Sehne wieder ihre ursprüngliche Struktur in Festigkeit und Elastizität zu ge-

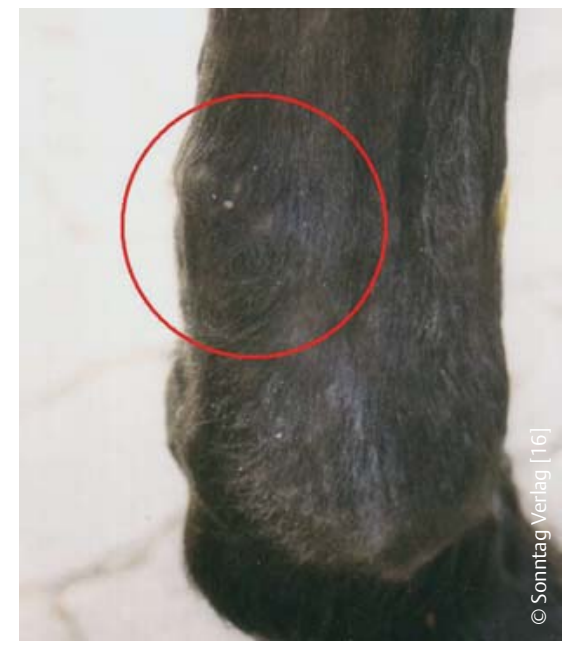

Abb. 5 Sehne zeigt nach insgesamt 5-wöchiger Therapie nochmals eine deutliche Reduktion der Schwellung und Schmerzhaftigkeit. Das Pferd geht lahmfrei.

ben. Hierbei wurde nach vorherigem ambulantem oder stationärem Therapiebeginn die Großzahl der Behandlungen vom Besitzer zu Hause durchgeführt. Auch die Therapiedauer war deutlich höher, 2-12 Wochen (im Mittel 5,3 Wochen). Die Diskrepanz ist aber in Bezug zu der sehr viel höheren Behandlungszahl deutlich kleiner. Dies zeigt, dass selbstverständlich neben dem Zeitraum, die die Sehne zur Regeneration benötigt, die intensive Behandlung mit dem Laser unabdingbar ist, um eine Ausheilung zu erreichen.

Die Grenzen der Möglichkeiten der Laserakupunktur zeigt eindeutig Patient Nr. 6, mit dem vollständigen Niederbruch der Sehnen. Da der Besitzer trotz der infausten Prognose die Therapie ausdrücklich wünschte, wurde der Therapieversuch für angemessen gehalten, zumal sich der Patient schon relativ gut mit dem Problem arrangiert hatte und sich in der Box vorsichtig bewegen konnte und auch guten Appetit zeigte. Da sich nach 4 Wochen keine Besserung ergab, musste er schließlich doch euthanasiert werden.

Auf der anderen Seite zeigen aber vor allem die Resultate der beiden Fallbeispiele, welches außerordentliche Potenzial die Laserakupunktur bietet.

\section{Diskussion}

Nach der empirischen Erfahrung des Autors in über 100 Praxisfällen akuter und chronischer Tendinitis im Zeitraum von 2005 bis 2013 konnte mit der lokalen La- 


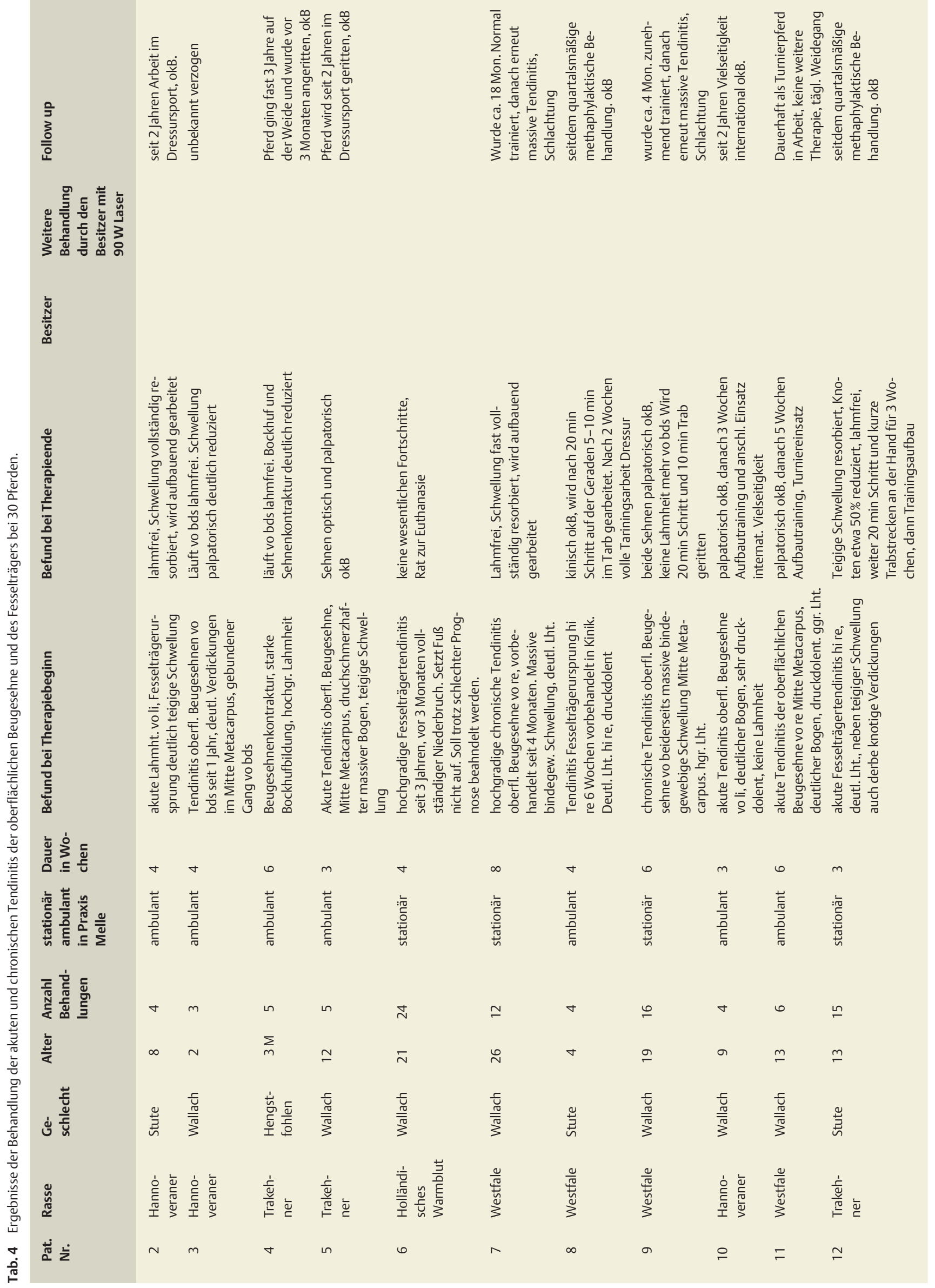




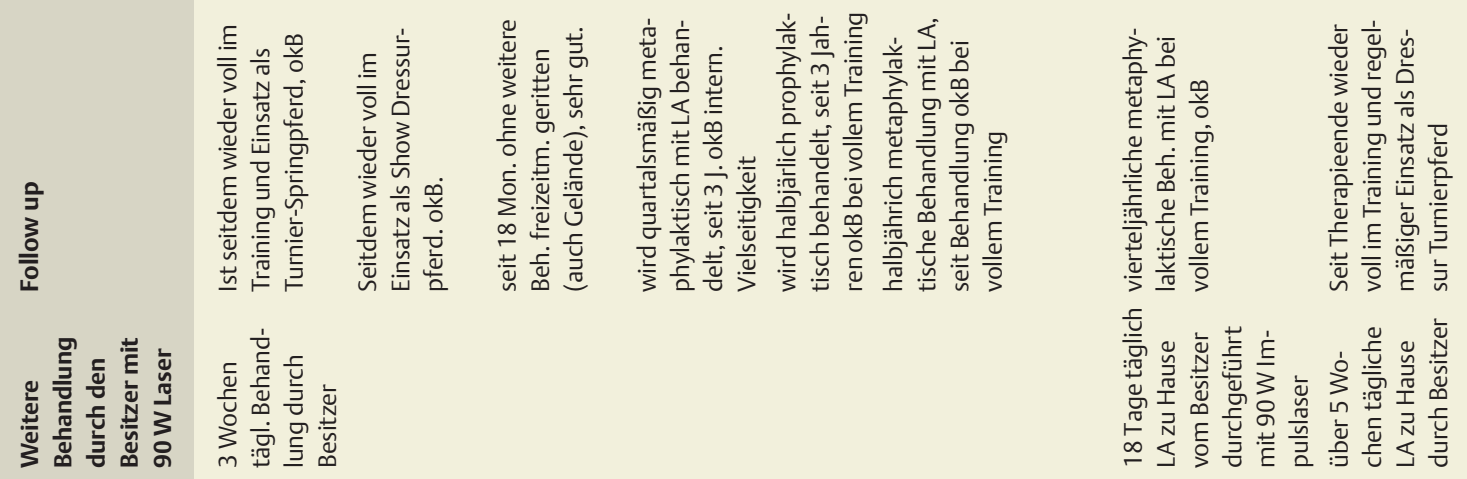

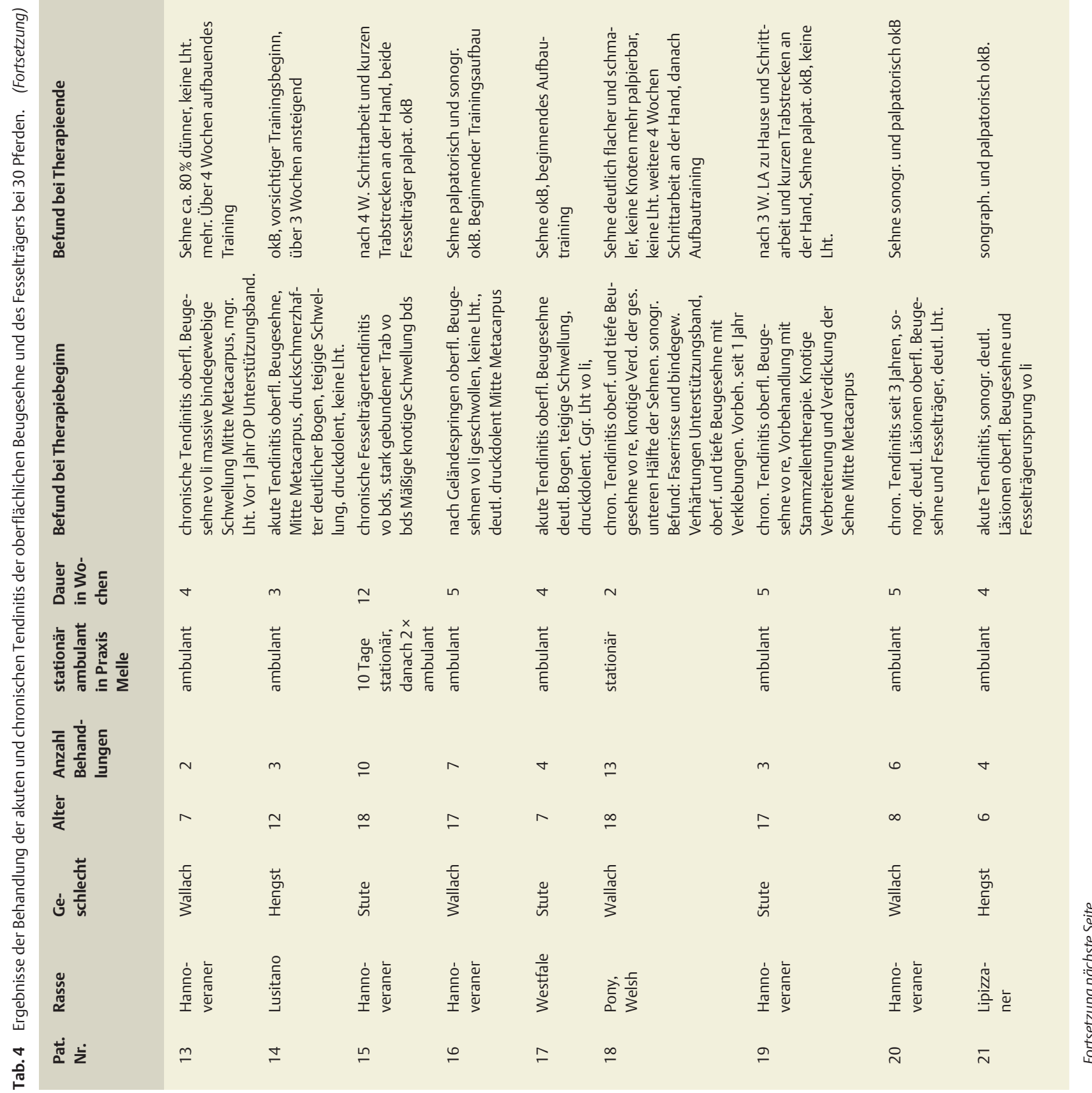




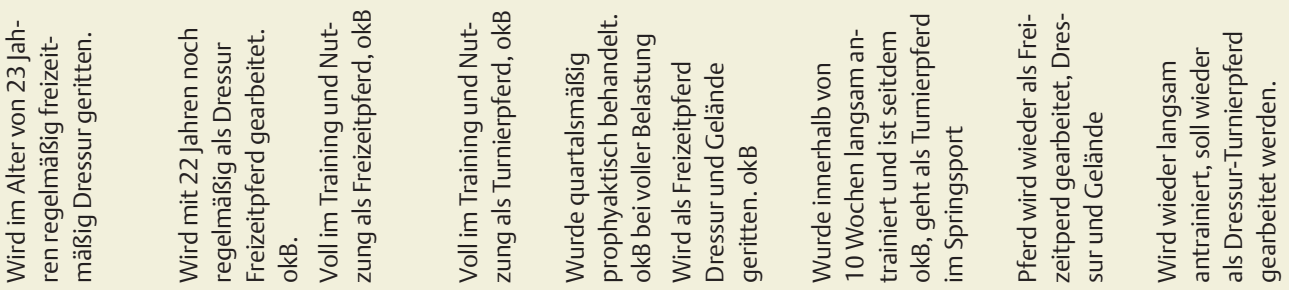

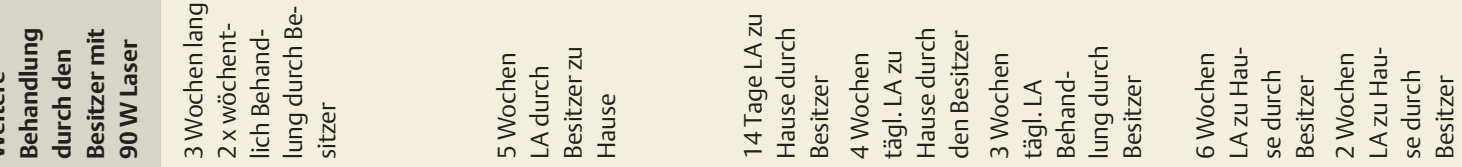

遂

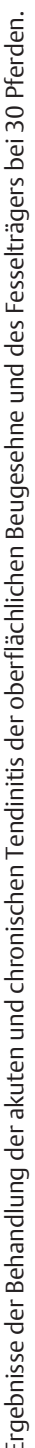

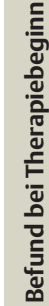

产

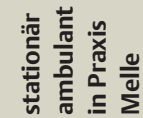

琣

亲

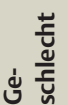

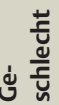

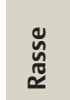

ํㅗㄴ

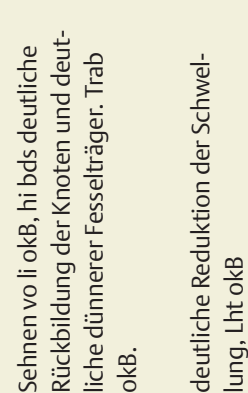

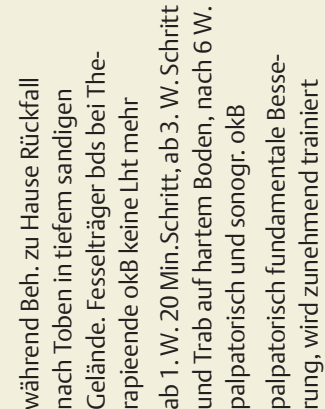

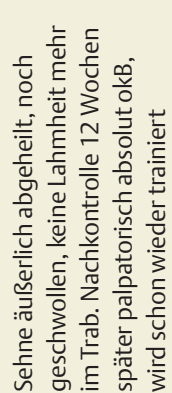

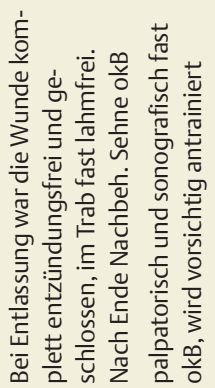

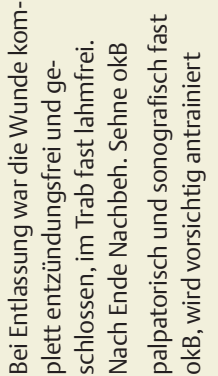

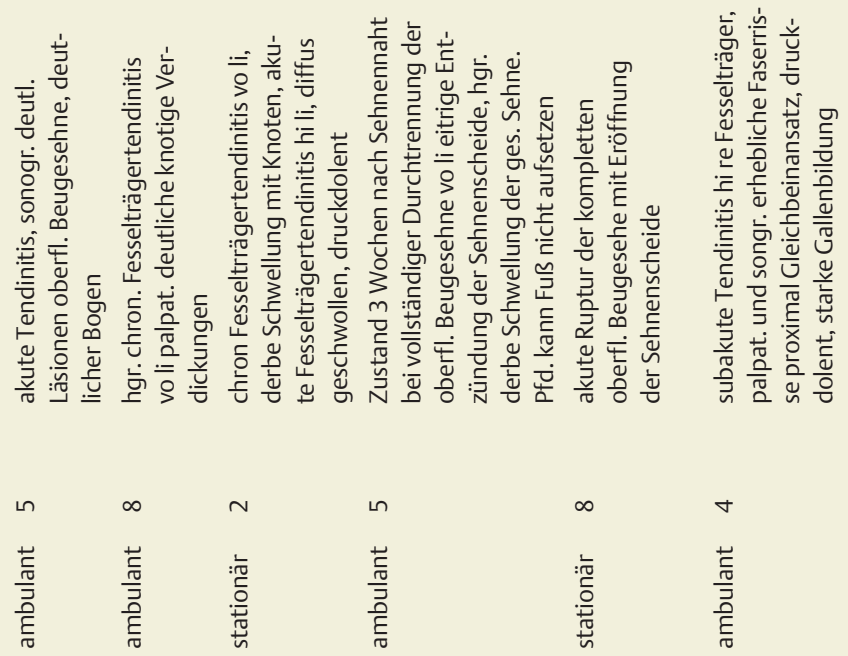

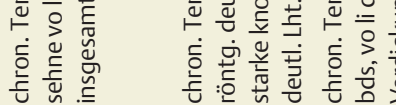

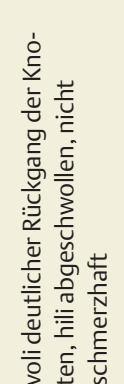

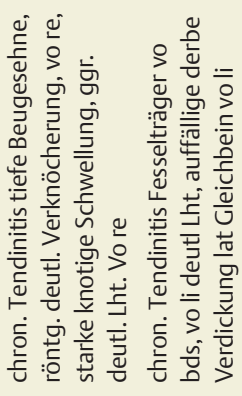
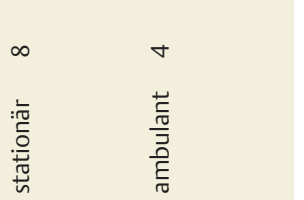

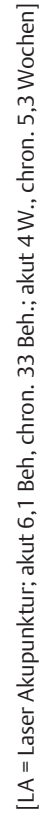


sertherapie in Verbindung mit Akupunktur selbst bei schon sehr fortgeschrittenen Fällen bei fast allen Patienten eine wesentliche Verbesserung der Sehnenstruktur und in den meisten Fällen sogar eine vollständige Wiederherstellung und dauerhafte Belastbarkeit der Sehne erreicht werden. Dies wurde anhand palpatorischer sowie sonografischer Sehnenbefunde und anhand von Verlaufskontrollen der Patienten festgestellt. Der Autor sieht es daher empirisch als deutlich erwiesen an, dass die Therapie mit geeigneten Impulslasern in der Lage ist, eine hohe antiinfektive, antiphlogistische, gewebsdemarkierende und regenerative Wirkung auf sogar massiv traumatisiertes Sehnengewebe auszuüben. Die lokale Wirkung des Lasers wird dabei erheblich durch die vegetativ regulierende und das Immunsystem unterstützende Wirkung der Akupunktur ergänzt.

\section{Schlussfolgerungen}

Es wäre sehr wünschenswert, vor allem im Interesse der betroffenen Patienten und deren Besitzer, wenn der oben beschriebenen Therapie von equinen Tendopathien in Zukunft die Beachtung geschenkt wird, die sie verdient. Die dargestellten Ergebnisse der beiden extremen Fallbeispiele zeigen, dass die Laserakupunktur in dieser konsequenten Form eine hervorragende Option für die Behandlung nahezu aller Formen der akuten und chronischen Tendinitis beim Pferd darstellt.

Auch wenn im Rahmen der Evidenz-basierten Medizin der Kasuistik, der Erfahrung und der empirischen Untersuchung ein sehr hoher Stellenwert eingeräumt wird [20], wäre es sehr wünschenswert und Erfolg versprechend, wenn diese Methode unter geeigneteren reproduzierbaren Bedingungen weiter untersucht und verifiziert werden könnte. $\bullet$

\section{Summary}

Treatment of acute and chronic equine tendonitis with local laser therapy and acupuncture: A study of $\mathbf{3 0}$ horses

In this study, 30 horses with acute and chronic tendinitis of the superficial digital flexor tendon and the suspensory that were treated solely with laser acupuncture are presented. Based on 2 case studies, the efficacy of laser acupuncture using a
$904 \mathrm{~nm}$ gallium arsenide laser is demonstrated in horses with completely transected superficial tendon. The importance of choosing the optimal laser is highlighted and specifically the application of $904 \mathrm{~nm}$ pulse laser with 90 watts peak pulse power is given more preference. This is due to its physical properties which include the high penetration depth in traumatized tissue without causing thermal reaction in the tissues. This laser produces an excellent healing tendency in the traumatized tendon tissue through direct ATP provision, high peroxide clearance, restructuring of the connective tissue texture and increase in the production of collagen fibers. In addition to topical laser therapy, appropriate acupuncture points are stimulated to improve tissue demarcation (TH5), for improvement of connective tissue structure and collagen fiber synthesis (SP2), stimulation of the immune system (SP4 and TH5) and anti-inflammatory therapy (GB41). All these points are stimulated with the same laser. In addition to laser acupuncture, no adjuvant therapies such as antibiotics or anti-inflammatory drugs were used. This therapy is recognized in acute and chronic tendinitis as a very effective method of healing.

\section{(Dey words}

904 nm impulse laser - acupuncture - superficial flexor tendon - tendinitis - horse

\section{Literatur}

Literatur ist in der Online-Version unter www.thieme-connect.de/products einsehbar.

Online zu finden unter

http://dx.doi.org/10.1055/s-0035-1568191

Dr. Uwe Petermann

Praktischer Tierarzt, Akupunktur

Schmale Str. 20, 49326 Melle

Tel.: $05428 / 93003$

E-Mail: druwepetermannmelle@t-online.de www.akupunkturtierarzt.de

Geb. 1953, 1974-1979 Studium der Tiermedizin in Hannover; Promotion 1980; Assistenzzeit an der Tierklinik Wahlstedt; seit 1982 eigene Praxis; seit 1995 reine Akupunkturpraxis, seit 1997 regelmäßige Durchführung eigener Akupunkturkurse für Tierärzte sowie weltweite Vorträge. Derzeitiger Präsident der IVAS. President Elect der International Veterinary Acupuncture Society. 